\title{
Higher long-term visit-to-visit glycemic variability predicts new-onset atrial fibrillation in patients with diabetes mellitus
}

\author{
Jung-Chi Hsu ${ }^{1,2,3}$, Yen-Yun Yang ${ }^{4}$, Shu-Lin Chuang ${ }^{4}$, Chih-Chieh Yu² and Lian-Yu Lin ${ }^{2 *}$ (i)
}

\begin{abstract}
Background: Atrial fibrillation (AF) is prevalent in patients with type 2 diabetes mellitus (T2DM). Glycemic variability (GV) is associated with risk of micro- and macrovascular diseases. However, whether the GV can increase the risk of AF remains unknown.

Methods: The cohort study used a database from National Taiwan University Hospital, a tertiary medical center in Taiwan. Between 2014 and 2019, a total of 27,246 adult patients with T2DM were enrolled for analysis. Each individual was assessed to determine the coefficients of variability of fasting glucose (FGCV) and $\mathrm{HbA1C}$ variability score (HVS). The GV parameters were categorized into quartiles. Multivariate Cox regression models were employed to estimate the relationship between the GV parameters and the risk of AF, transient ischemic accident (TIA)/ischemic stroke and mortality in patients with T2DM.
\end{abstract}

Results: The incidence rates of AF and TIA/ischemic stroke were 21.31 and 13.71 per 1000 person-year respectively. The medium follow-up period was 70.7 months. In Cox regression model with full adjustment, the highest quartile of FGCV was not associated with increased risk of AF [Hazard ratio (HR): 1.12, 95\% confidence interval (Cl) 0.96-1.29, $\mathrm{p}=0.148]$ or TIA/ischemic stroke ( $\mathrm{HR}: 1.04,95 \% \mathrm{Cl} 0.83-1.31, \mathrm{p}=0.736$ ), but was associated with increased risk of total mortality (HR: 1.33, 95\% Cl 1.12-1.58, $\mathrm{p}<0.001$ ) and non-cardiac mortality (HR: 1.41, 95\% Cl 1.15-1.71, $\mathrm{p}<0.001)$. The highest HVS was significantly associated with increased risk of AF (HR: 1.29, 95\% Cl 1.12-1.50, p<0.001), total mortality (HR: 2.43, 95\% Cl 2.03-2.90, $\mathrm{p}<0.001$ ), cardiac mortality ( $\mathrm{HR}: 1.50,95 \% \mathrm{Cl} 1.06-2.14, \mathrm{p}=0.024$ ) and non-cardiac mortality (HR: $2.80,95 \% \mathrm{Cl} 2.28-3.44, \mathrm{p}<0.001$ ) but was not associated with TIA/ischemic stroke (HR: 0.98, 95\% Cl 0.78-1.23, $\mathrm{p}=0.846$ ). The Kaplan-Meier analysis showed significantly higher risk of AF, cardiac and non-cardiac mortality according to the magnitude of GV (log-ranktest, $p<0.001$ ).

Conclusions: Our data demonstrate that high GV is independently associated with the development of new-onset AF in patients with T2DM. The benefit of maintaining stable glycemic levels to improve clinical outcomes warrants further studies.

Keywords: Glycemic variability, Atrial fibrillation, Diabetes mellitus

\footnotetext{
*Correspondence: ccyu2014@ntu.edu.tw; hspenos@gmail.com

${ }^{2}$ Division of Cardiology, Department of Internal Medicine, National

Taiwan University College of Medicine and Hospital, No.7, Chung Shan South Road, 100 Taipei, Taiwan

Full list of author information is available at the end of the article
}

\begin{abstract}
Introduction
Atrial fibrillation (AF) is prevalent in patients with aging, congestive heart failure (CHF), hypertension $(\mathrm{HTN})$, and diabetes mellitus (DM). Patients with type 2 diabetes mellitus (T2DM) carry an overall $35 \%$ higher risk of AF compared to general population, and increased blood glucose has a dose-response
\end{abstract} permits use, sharing, adaptation, distribution and reproduction in any medium or format, as long as you give appropriate credit to the original author(s) and the source, provide a link to the Creative Commons licence, and indicate if changes were made. The images or other third party material in this article are included in the article's Creative Commons licence, unless indicated otherwise in a credit line to the material. If material is not included in the article's Creative Commons licence and your intended use is not permitted by statutory regulation or exceeds the permitted use, you will need to obtain permission directly from the copyright holder. To view a copy of this licence, visit http://creativecommons.org/licenses/by/4.0/. The Creative Commons Public Domain Dedication waiver (http://creativeco mmons.org/publicdomain/zero/1.0/) applies to the data made available in this article, unless otherwise stated in a credit line to the data. 
relationship with the incidence of AF [1, 2]. However, whether DM itself is an independent risk for AF or diabetic environment prone to AF has been controversial. Potential proarrhythmic mechanisms linking AF to DM are complex. Interstitial fibrosis and atrial dilatation, marker of DM atriopathy can cause structural remodeling. Decreased sodium ion channel, intracellular calcium dysregulation, and abnormal expression of connexins from connexin-40 to connexin-43, can change atrial refractoriness, resulting in electrical remodeling. Autonomic imbalance, sympathetic overactivation, and heterogeneous distribution of sympathetic nerves lead to autonomic remodeling $[3,4]$.

Apart from focusing primarily on measurement of fasting plasma glucose (FPG), hemoglobin A1c (HbA1c), advanced glycation end products (AGEs), glucagon-like peptide 1 (GLP-1), short-term glycemic variability within-days or months or even years have been considered as important risk factors for cardiovascular disease [5]. Glycemic fluctuation has been shown to over-activate oxidative stress and inflammation system, aggravating greater vascular damage and cardiomyopathy than that in chronic stable hyperglycemia [5, 6]. Increased glycemic variation (GV) also has adverse effect on autonomic function and increases the thrombotic properties of the platelets, which may be associated with higher incidence of major adverse cardiovascular event (MACE) [7, 8].

Previous studies have shown that patients with higher acute GV have more vulnerable plaques and poorer prognosis of acute coronary syndrome [9-11]. Shortterm GV is also associated with increased mortality after cardiac procedure such as transcatheter aortic valve implantation [12]. Long-term GV was found to be associated with greater progression of coronary artery calcification in young adults [13]. High GV also causes left ventricular diastolic dysfunction and increased the risk of heart failure [14, 15]. In the Antihypertensive and Lipid-Lowering Treatment to Prevent Heart Attack Trial (ALLHAT) study, GV was independently associated with the risks of cardiovascular event and all-cause mortality [16].

Nevertheless, the majority of the studies regarding the effect of GV have focused on diabetic macro- and micro-vascular complications. Whether GV is associated with the development of AF is not known. Investigating the contribution of GV on the development of AF may advance our understanding of how dysfunction in glucose homeostasis impacts atrial remodeling. In this cohort study, we plan to investigate the association of long-term GV with the incidence of AF and related cardiovascular outcomes in a group of patients with T2DM.

\section{Methods}

\section{Study population and data collection}

The study was approved by the Institutional Review Board (IRB) of National Taiwan University Hospital. The study database was from National Taiwan University Hospital integrated Medical Database (NTUH-iMD) which was composed of detailed medical information from a tertiary medical center in Taiwan. Since AF is prevalent in patients with older age, we enrolled those with age above 50 years and diagnosed with T2DM at the National Taiwan University Hospital from January 1, 2014, to December 31, 2019. Patients with previous AF or lost to follow-up (defined as an absence of followup at the outpatient clinics between Oct. 1st to Dec. 31, 2019 since we followed patients at least every three months) were excluded. For simplicity, patients who had severe end organ damage including history of congestive heart failure (CHF) or above stage 3 chronic kidney disease (CKD) were also excluded. Baseline characteristics including body mass index (BMI), hypertension (HTN), hyperlipidemia, coronary artery disease (CAD), acute coronary syndrome (ACS), myocardial infarction (MI), chronic obstructive pulmonary disease (COPD), peripheral arterial occlusive disease (PAOD) were obtained from the electronic health records (EHRs). Estimated glomerular filtration rate (eGFR) was calculated by modification of diet in renal disease (MDRD) equation. Prescription information were categorized into antiarrhythmic agents, calcium channel blocker (CCB), betablocker, angiotensin converting enzyme inhibitor (ACEI), angiotensin receptor blocker (ARB), mineralocorticoidreceptor antagonist (MRA), anticoagulants including direct oral anticoagulant (DOAC) and warfarin, and anti-diabetic medications including insulin, metformin, sodium-glucose co-transporter-2 (SGLT2) inhibitor, dipeptidyl peptidase 4 (DPP4) inhibitor, sulphonylurea, repaglinide, acarbose, thiazolidinedione (TZD), glucagon like peptide-1 (GLP-1) agonist. Echocardiographic studies were performed with Phillips iE33 (Phillips, Bothell, WA, USA) and two-dimensional-guided M-mode measurements with a $3.0-$ or $3.5-\mathrm{MHz}$ transducer. Left atrium (LA) size, LV internal dimension in end-diastole (LVIDd) and systole (LVIDs), and LV ejection function were collected in the parasternal long-axis view with M-mode cursor. LA size was anterior-posterior diameter measured at the end-ventricular systolic phase. LV mass was calculated by using the Devereux formula. All the echocardiographic data were assessed from the EHRs.

The outcomes were AF, transient ischemic accident (TIA), ischemic stroke and mortality. Death events were also adjudicated by a central committee, and were separated to cardiac and noncardiac mortality. AF and its occurrence time were identified by the diagnosis code 
from either the EHRs or the standard 12-lead electrocardiogram. The index dates of outcomes were defined as the dates of diagnosis. All medical records were reviewed until their last clinical visit or death.

\section{Glycemic variability measurement}

We calculated two measures of GV for each individual: the coefficient variability of the mean FPG (FGCV) and the HbA1c variability score (HVS). FPG and HA1C were measured quarterly at visit-to-visit outpatient department. FPG was measured in subjects who reported fasting more than $8 \mathrm{~h}$. The calculation of FPG and HbA1c variability was from at least three successive measurements. Only patients having both FGCV and HVS were included for analysis. FGCV (\%) was calculated as the standard deviation (SD) of FPG divided by the mean FPG, and then divided by the square root of the ratio of FPG measurements $\mathrm{n}$ to $\mathrm{n}-1(\sqrt{n /(n-1)})$ to account for the influence of FPG measurement number $[17,18]$. HVS is the number of measures within an individual where the $\mathrm{HbA} 1 \mathrm{c}$ has changed by $>0.5 \%(5.5 \mathrm{mmol} / \mathrm{mol})$ from the prior value, as a percentage of the total number of HbA1c measures between the diagnosis of diabetes and the outcome. In brief, HVS is a percentage of HbA1c fluctuation events $(\Delta>0.5 \%)$ [19].

\section{Statistical analysis}

Patients were categorized into four groups according to the quartiles of FGCV or HVS. Continuous variables were described as mean (SD) and categorical variables were reported as frequency (percentage). Differences among groups were tested by using chi square test for categorical variables and analysis of variance (ANOVA) test for continuous variables. Multivariate Cox proportional hazards models were constructed to assess the association of categorical and continuous measures. The relationship between GV and the development of diabetes complications was assessed by Cox regression from which hazard ratios (HRs) and 95\% confidence intervals (CIs) were derived. The assumption of proportionality was validated by verifying the Schoenfeld residuals. Trend analysis in the general linear model and CochraneArmitage test were used for evaluating dose-response effects.

The semiparametric Cox regression models were sequentially adjusted for the following baseline covariates. The model 1 was crude model without any adjustment. The model 2 adjusted for age, gender (male as reference group), baseline BMI, history of HTN, COPD, CAD, PAOD and prior TIA/ischemic stroke, baseline FPG, baseline HbA1c, and baseline estimated glomerular filtration rate (eGFR). The model 3 adjusted for model 2 plus three echocardiogram parameters including LA size,
LVEF, LVM. Model 4 adjusted for model 3 plus the medications including antiplatelet, anticoagulant, $\mathrm{CCB}$, betablocker, ACEI/ARB, diuretic, statin, insulin, metformin, SGLT2 inhibitor, DDP4 inhibitor, sulphonylurea, repaglinide, acarbose, TZD, GLP-1 agonist. Survival analyses were presented by using Kaplan-Meier curves and the significance of difference between curves were examined by log-rank test. All statistical analyses were performed using SAS statistical software package (version 9.4. SAS Institute Inc., Cary, NC, USA). A two-tailed p-value of less than 0.05 was considered statistically significant.

\section{Results}

\section{Baseline characteristics}

The flowchart of patient selection was demonstrated in Fig. 1. A total of 74,835 with T2DM diagnosis code between 2014 and 2019 were enrolled. Among them, 121 patients without firm evidence of T2DM (blood test, DM medications) and 1607 patients aged below 50 years were excluded. We excluded 1755 patients with pre-existing AF, 125 patients with severe CHF (ever hospitalization caused by acute decompensated heart failure), and 6054 patients with moderate or severe CKD ( $\geq$ stage 3 CKD). We only included patients with both FGCV and HVS data, so 38,628 patients who only had either FGCV or HVS data were excluded. We also excluded the patients with $\mathrm{FGCV}=0 \quad(\mathrm{n}=81)$ or $\mathrm{HVS}=0 \quad(\mathrm{n}=11,141$, over $70 \%<10$ measurements) to simplify the analysis and uniform the approach for FGCV and HVS. Finally, a total of 27,246 subjects were enrolled for further analysis. The patients were grouped according to the quartiles of either FGCV or HVS. The clinical, biochemical and anthropometric characteristics were presented in Table 1 . The subjects in the highest quartile of FGCV were older, had higher baseline FPG and HbA1c, had worse baseline eGFR, were more likely to have PAOD and less likely to have HTN or CAD. The subjects in the highest quartile of HVS were more male gender, had less HTN, had higher baseline FPG and HbA1c levels and lower baseline eGFR.

Over a median follow-up of 70.7 months, there were 2762 AF events (overall incidence rate 21.31 per 1000 person-year) and $1803 \mathrm{TIA} /$ ischemic stroke events (overall incidence rate 13.71 per 1000 person-year). The incidence rates of AF for FGCV quartiles were 16.47, 17.66, 19.86, and 31.76 per 1000 person-year. The incidence rates of AF for HVS quartiles were 14.19, 19.30, 24.37, and 29.27 per 1000 person-year. The incidence rates of $\mathrm{TIA} /$ ischemic stroke for FGCV quartiles were 11.98, $11.52,13.88,17.66$ per 1000 person-year. The incidence rates of TIA/ischemic stroke for HVS quartiles were $10.32,15.83,16.52,15.63$ per 1000 person-year. There were 3545 deaths in which 615 (17.35\%) were cardiac causes. 


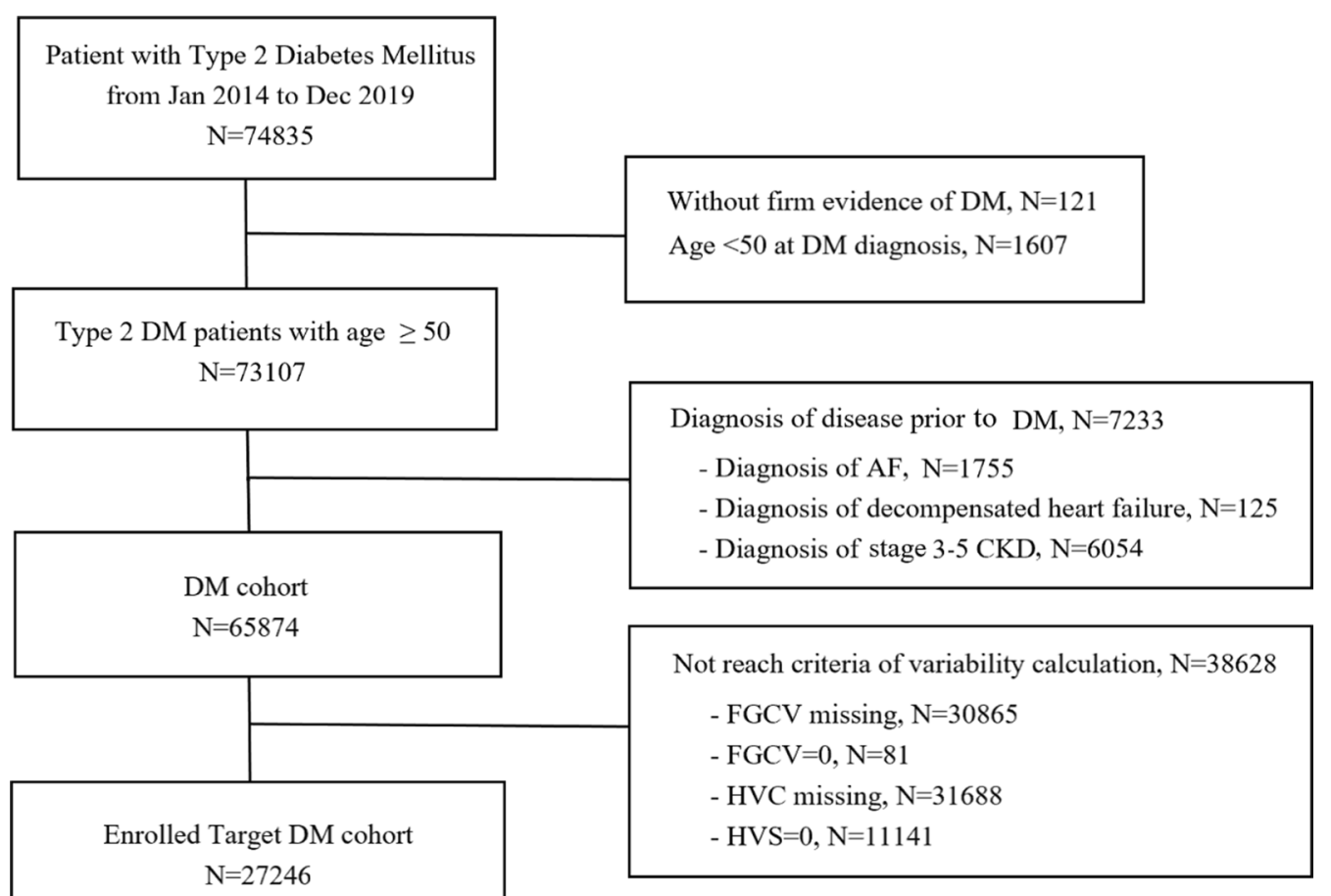

Fig. 1 Flow chart of patient selection

\section{Measures of glycemic variability and outcomes}

As shown in Table 2, in comparison with the first quartile of FGCV, the hazard ratios (HRs) across quartiles (second to fourth quartiles) for AF were 1.09 (95\% CI 0.97-1.23, $\mathrm{p}=0.137), 1.24$ (95\% CI 1.10-1.39, $\mathrm{p}<0.001$ ), and 2.00 (95\% CI 1.80-2.22, $\mathrm{p}<0.001)$ in model 1 without adjustment. After model 2 and 3 adjustment, the fourth quartile remained significantly associated with the development of AF and the HRs were 1.74 (95\% CI 1.55-1.96, $\mathrm{p}<0.001)$ and 1.45 (95\% CI 1.26-1.66, $\mathrm{p}<0.001)$ for model 2 and 3 respectively. In fully adjusted model 4 , the HRs for AF became insignificant $(1.12$, 95\% CI $0.96-$ $1.29, \mathrm{p}=0.148)$. As for other outcomes, in fully adjusted model, the fourth quartile remained significant for total mortality $(1.33,95 \%$ CI $1.12-1.58, \mathrm{p}<0.001)$ and noncardiac mortality $(1.41,95 \%$ CI $1.15-1.71, \mathrm{p}<0.001)$ but became insignificant for TIA/ischemic stroke $(1.04,95 \%$ CI $0.83-1.31, \mathrm{p}=0.736)$ and cardiac mortality $(1.05,95 \%$ CI $0.72-1.52, \mathrm{p}=0.810$ ).

Also shown in Table 2, by using the first quartile of HVS as reference, the HRs for AF remained significant in the third $(1.17,95 \%$ CI $1.02-1.34, \mathrm{p}=0.024)$ and fourth quartile $(1.29,95 \%$ CI $1.12-1.50, \mathrm{p}<0.001)$ in fully adjusted model. As for other outcomes, in fully adjusted model, in comparison with the first quartile, all three quartiles were significantly associated with total mortality and non-cardiac mortality but only the fourth quartile remained significant for cardiac mortality $(1.50,95 \% \mathrm{CI}$ $1.06-2.14, \mathrm{p}=0.024)$. For TIA/ischemic stroke, all quartiles were nonsignificant in fully adjusted model. Forest plot of HRs in fully adjusted models were demonstrated in Fig. 2A (FGCV) and 2B (HVS). The numbers of FPG and $\mathrm{HbA} 1 \mathrm{c}$ tests during follow-up were presented in Additional file 1).

The results of Kaplan-Meier analysis were demonstrated in Fig. 3. As shown in Fig. 3, the probability of AF (3A), mortality (3B), non-cardiac mortality (3C) and cardiac mortality (3D) were significantly different across quartiles of HVS (All log-rank $\mathrm{p}<0.001$ ).

\section{Discussion}

Our study showed that a greater GV is associated with a higher incidence of AF in patients with type $2 \mathrm{DM}$. In addition, a greater GV is independently associated with higher chances of cardiac and all-cause mortality. To our knowledge, this is the first cohort study to explore the association of long-term $\mathrm{GV}$ with the development of AF.

High GV has been proved to be associated with increased risk for cardiovascular events and poor prognosis [20, 21]. However, it's impact on arrhythmia has been seldom studied. In a large Korea cohort of healthy population, the metabolic variability score composed of glucose level, blood pressure, total cholesterol level, and BMI showed a close association with the risk of $\mathrm{AF}$, and the 


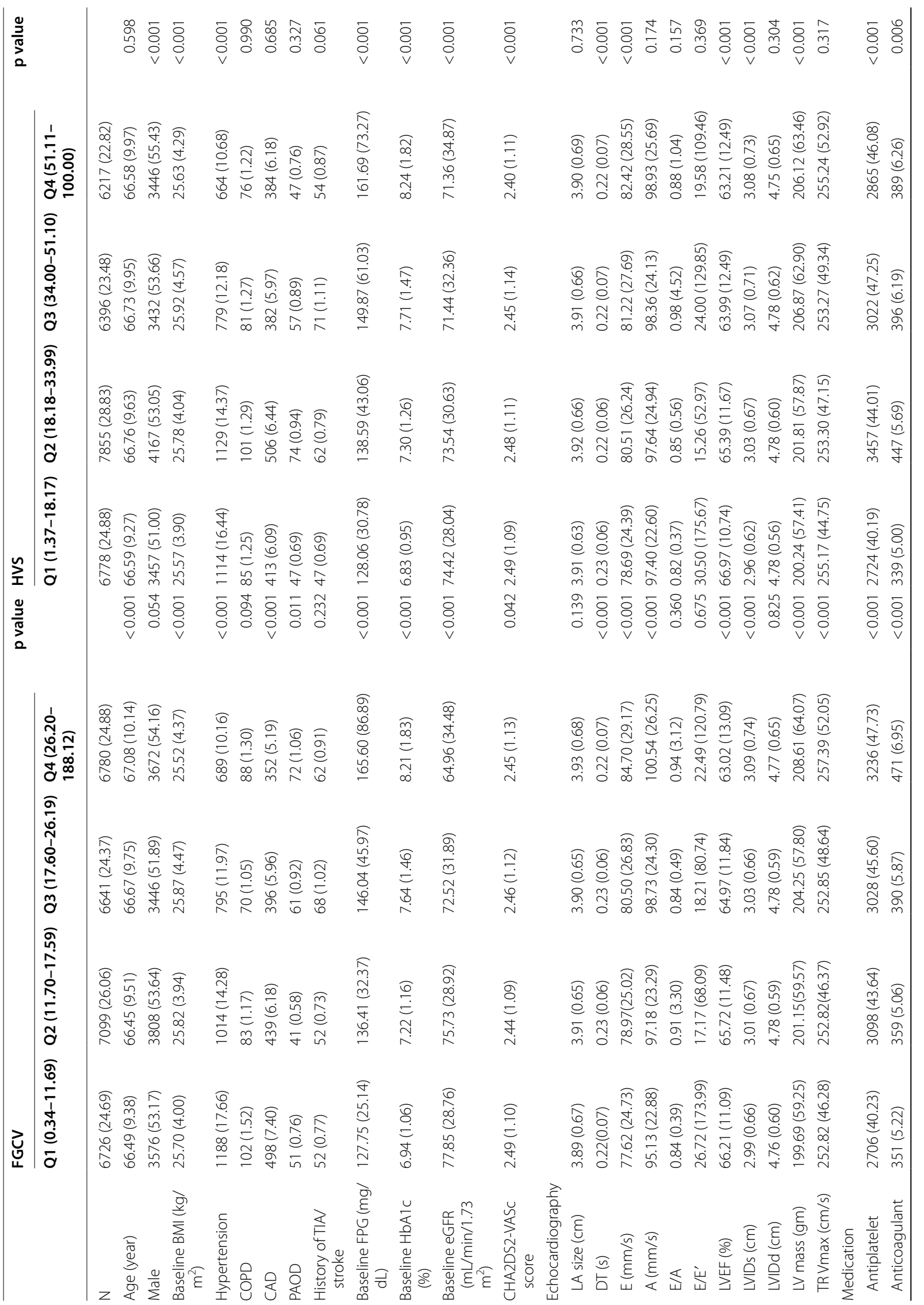




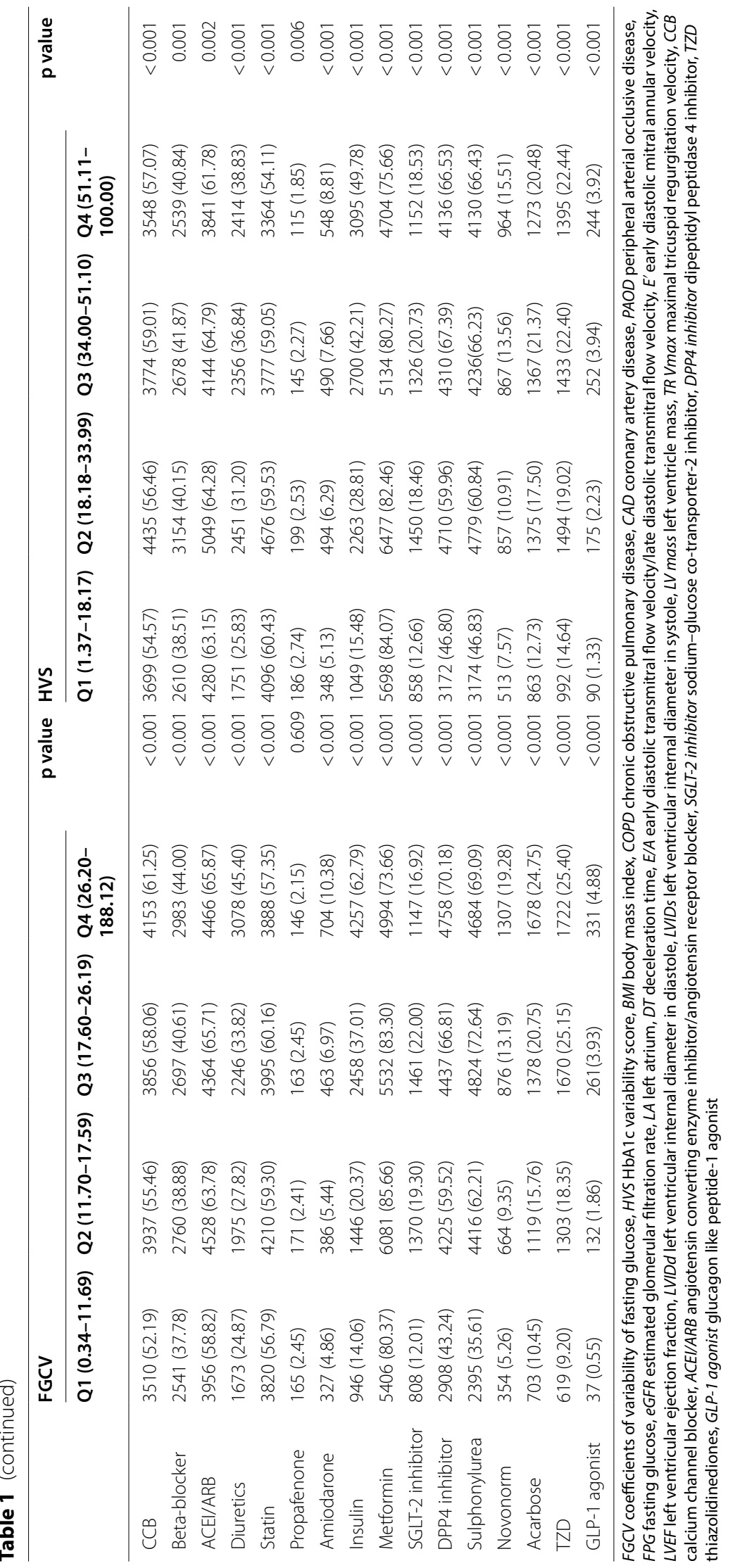




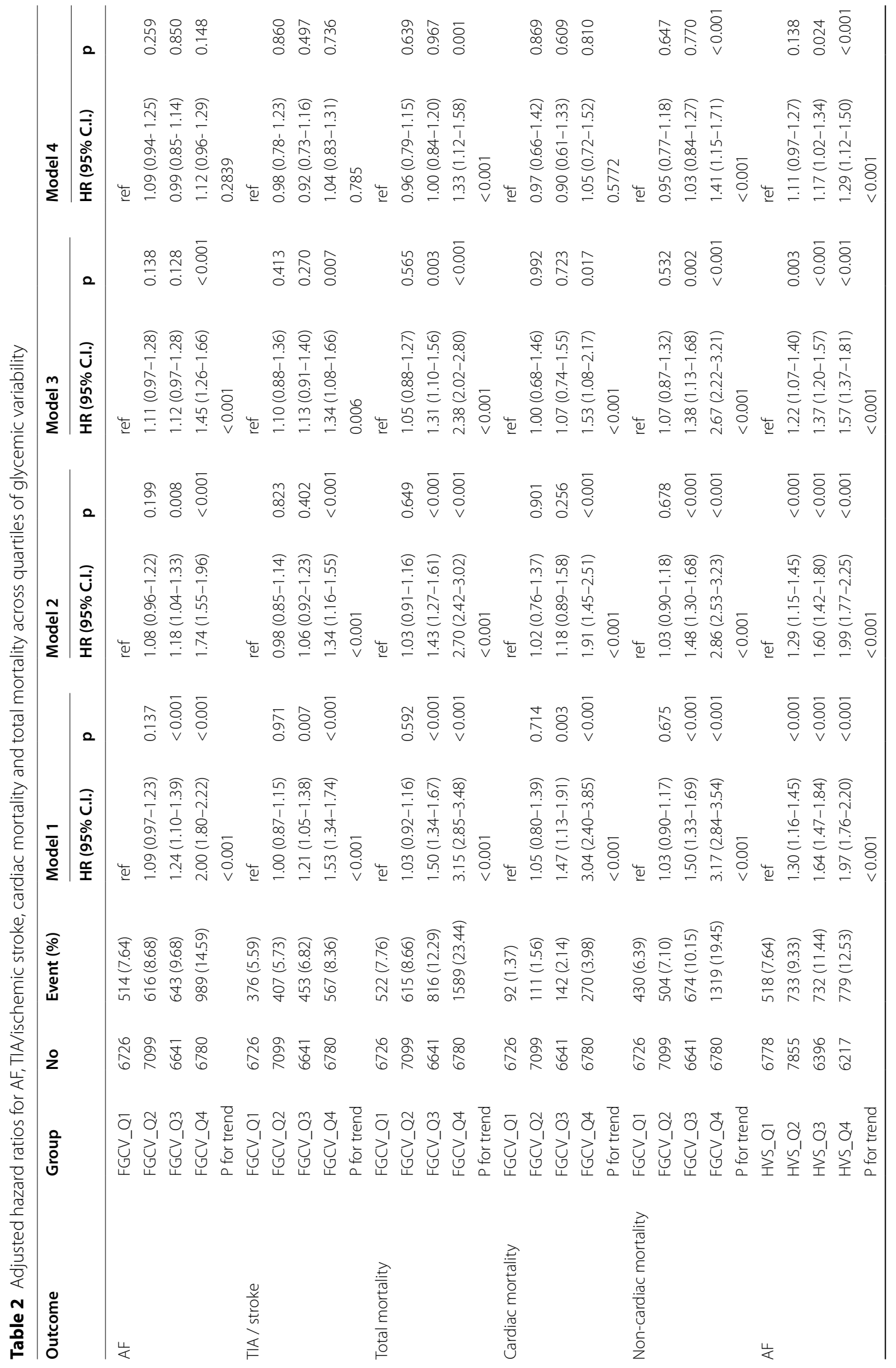




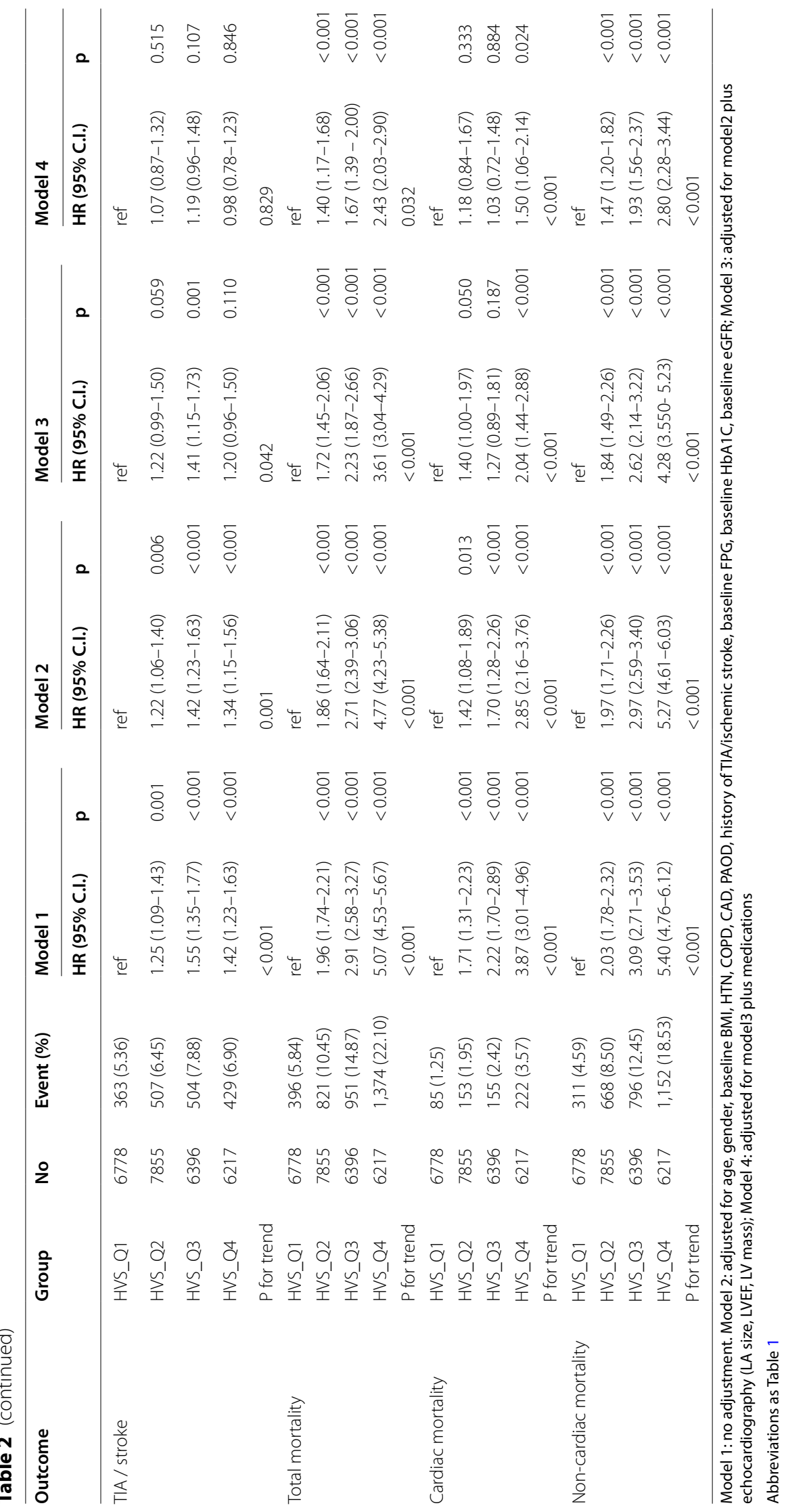




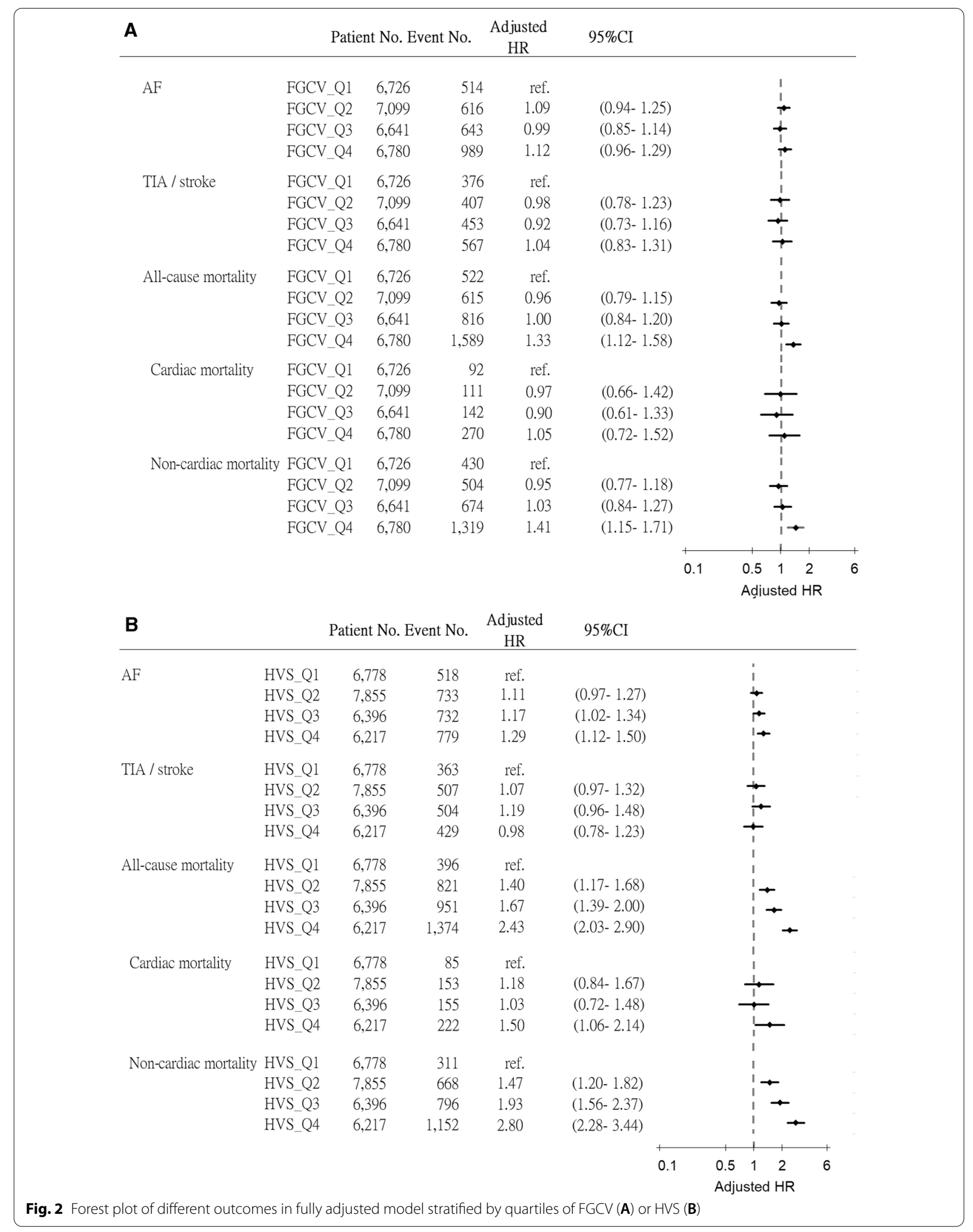



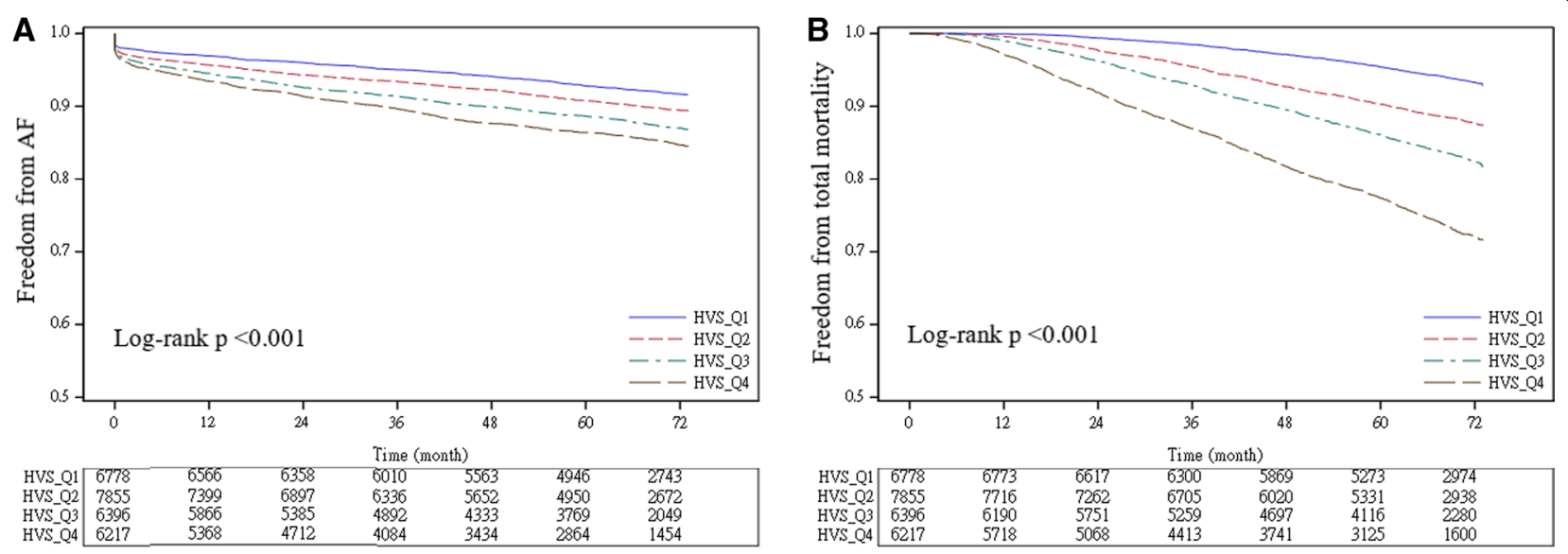

c

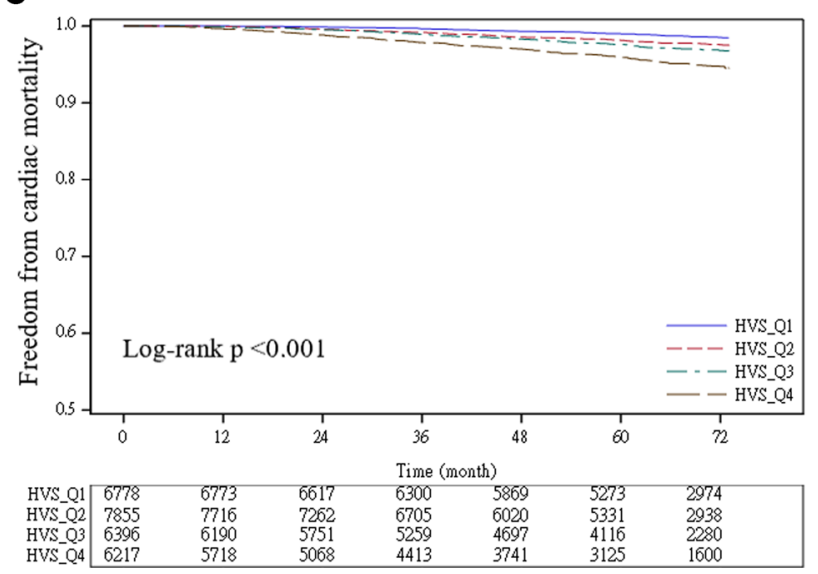

D

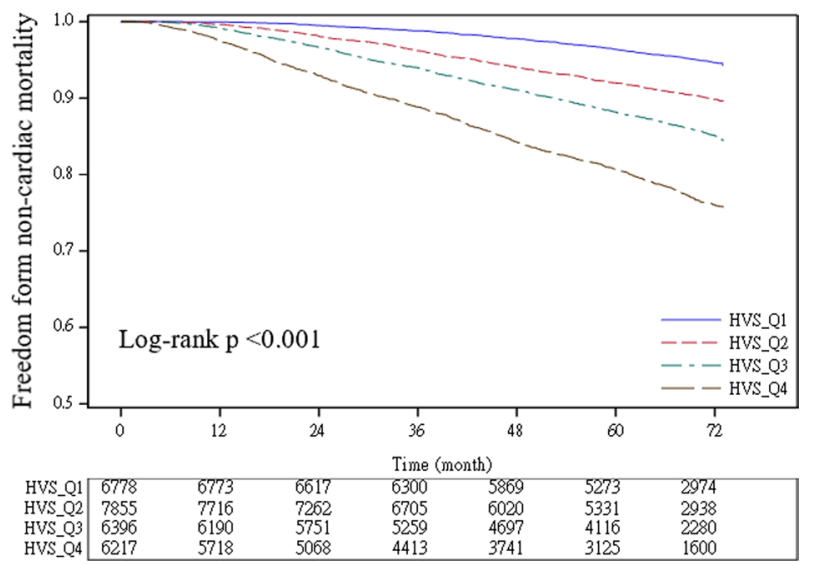

Fig. 3 Kaplan-Meier analysis stratified by HVS for different outcomes: AF (A),

A), total death (B), cardiac death $(\mathbf{C})$ or non-cardiac death (D)

incidence was about 0.8 to 1.2 per 1000 person-year [22]. In our T2DM cohort, the overall incidence of AF was 21.31 per 1000 person-year, which was apparently much higher than the Asian healthy population without DM. The pathophysiology of AF development in DM has not been elaborately investigated. In a diabetic mice model, increased AF susceptibility was associated with reduced atrial conduction velocity, action potential duration prolongation, increased heterogeneity in repolarization, and increased interstitial atrial fibrosis [23]. In addition to blood sugar level, increased magnitude of GV may generate more reactive oxygen species than hyperglycemia alone. In diabetic rats, glucose fluctuations promote cardiac fibrosis by altering AKT signaling pathway and upregulate Txnip and $\mathrm{NADPH}$ oxidase expression which produce more reactive oxygen species levels, thereby increasing the incidence of $\operatorname{AF}[24,25]$. Other than direct effect, high GV may contribute to cardiac autonomic neuropathy which has a strong influence on cardiac arrhythmias [26].

Detrimental effects of glycemic fluctuations for AF are not only related to hyperglycemia but also hypoglycemia.
Acute hypoglycemia is associated with the initiation and perpetuation of AF via mechanisms of increasing sympathetic activation, catecholamine surge and inflammation. Failure of intensive glycemic control to prevent newonset AF in diabetic patients in the ACCORD trial hinted a potential harm that might come with hypoglycemia [27, 28]. Although the evidence for AF risk of hypoglycemia is circumstantial, a multitude of case reports of cardiac arrhythmias and electrocardiographic changes provoked by hypoglycemia had been reported [29].

The clinical meanings of the long-term and short-term GVs are different. Long-term GV is derived based on visit-to-visit measurements of HbA1c and FPG and is a marker of ambient hyperglycemia. In contrast, shortterm GV represents episodes of either hyperglycemia or hypoglycemia within days [30]. In our study, we observed that HVS derived from HbA1c has better consistency and performance than FGCV derived from FPG in predicting future events. This implies that the impact of GV on AF development is a long-term cumulative process. 
Long-term visit-to-visit HbA1c variability has been proved to be a strong predictor for both microvascular and macrovascular diseases and also for all-cause mortality [31, 32]. There are several ways to evaluate the HbA1c variability. One study showed that among the HbA1c variability parameters including mean of $\mathrm{HbAlc}$, yearly mean HbA1c, HbA1c-SD, HbA1c-CV and HVS, HVS performed the best in predicting microvascular events [33]. One reason is that many of the HbA1c variability parameters are affected by the mean HbA1c value. For example, since the mean $\mathrm{HbA} 1 \mathrm{c}$ is the denominator of the $\mathrm{CV}$, intensive DM treatment may lower the mean value while increase this variability index [34]. HVS is defined as a percentage of $\mathrm{HbA1c}$ fluctuation events and is relatively insensitive to the change of the HbA1c absolute value and thus can independently provide accurate and stable GV information [19]. Our study also identified some patient characteristics that are subjective to high GV including male gender, high BMI, high baseline HbA1c and CKD. These factors could be an important reference for physicians who take care of patients with DM.

\section{Limitations}

First, there might be surveillance bias because we did not screen for AF using a standardized, dedicated protocol at baseline or during follow-up period in this observative cohort. A recent study showed that the prevalence of asymptomatic, silent AF was $5-20 \%$ in diabetic subjects aged more than 65 years [35]. Since our patients were aged above 50 with a mean CHA2DS2-VASc score 2.56, the incidence of new-onset AF was possible to be underestimated. However, on the contrary, diabetic patients are more likely to have close follow-up, and thus they might have increased likelihood of being diagnosed with AF. The magnitude of these effects were difficult to be adjusted in this study. Second, we did not test all the reported GV parameters, such as average successive variability, average real variability of FPG, mean amplitude of glycemic excursion. We chose FGCV and HVS since they were commonly used, easily calculated parameters that could help physicians quickly determine the GV of their patients. Third, we excluded patients with severe end-organ damage including $\mathrm{CHF}$ and CKD to avoid complex AF confounders existing in these medical conditions. Whether the conclusion can be extrapolated to these conditions need further confirmation. Fourth, some known AF contributing factors, such as sleep apnea or sedentary behavior were not comprehensively evaluated in this study. Fifth, we excluded subjects who were not consistently followed at our out-patient clinics since the outcomes might be missing. This approach might cause selection bias but it could make sure that all the outcomes were accurately determined. Finally, this was a retrospective cohort study and the causal relationship might be less convincing.

\section{Conclusions}

Our data demonstrated that high GV is independently associated with the development of new-onset AF in patients with T2DM. The benefit of maintaining stable glycemic levels to improve clinical outcomes warrants further studies (Additional file 1).

\begin{abstract}
Abbreviations
AF: Atrial fibrillation; T2DM: Type 2 diabetes mellitus; GV: Glycemic variability; FGCV: Coefficients of variability of fasting glucose; HVS: HbA1c variability score; BMI: Body mass index; COPD: Chronic obstructive pulmonary disease; CAD: Coronary artery disease; PAOD: Peripheral arterial occlusive disease; FPG: Fasting plasma glucose; eGFR: Estimated glomerular filtration rate; LA: Left atrium; DT: Deceleration time; E/A: Early diastolic transmitral flow velocity/ late diastolic transmitral flow velocity; E': Early diastolic mitral annular velocity; LVEF: Left ventricular ejection fraction; LVIDd: Left ventricular internal diameter in diastole; LVIDs: Left ventricular internal diameter in systole; LV mass: Left ventricle mass; CCB: Calcium channel blocker; ACEI/ARB: Angiotensin converting enzyme inhibitor/angiotensin receptor blocker; SGLT-2 inhibitor: Sodiumglucose co-transporter-2 inhibitor; DPP4 inhibitor: Dipeptidyl peptidase 4 inhibitor; TZD: Thiazolidinediones; GLP-1 agonist: Glucagon like peptide-1 agonist.
\end{abstract}

\section{Supplementary Information}

The online version contains supplementary material available at https://doi. org/10.1186/s12933-021-01341-3.

Additional file 1. Supplementary data. Figure S1. Numbers of HbAc tests during follow-up. Figure S2. Numbers of fasting plasma glucose (FPG) tests during follow-up. Table S1. Median, mean, standard deviation (SD), minimum and maximum interval times (days) between $\mathrm{HbA} 1 \mathrm{c}$ and FPG (fasting plasma glucose) tests.

\section{Acknowledgements}

The authors would like to express their thanks to the staff of Department of Medical Research for providing clinical data from National Taiwan University Hospital-integrated Medical Database (NTUH-iMD).

\section{Authors' contributions}

LY-L contributed to the conception or design of the work. YY-Y and SL-C contributed to the acquisition, analysis of data for the work. JC-H interpreted and drafted the manuscript. CC-Y and LY-L critically revised the manuscript. All authors read and approved the final manuscript.

\section{Funding}

This research received no specific grant from any funding agency in the public, commercial, or not-for-profit sectors.

\section{Availability of data and materials}

The datasets used in this study were only available in the National Taiwan University Hospital. The SAS programs (codes) involved for this study are available from the corresponding author on reasonable request.

\section{Declarations}

\section{Ethics approval and consent to participate}

The study protocol complies with the Declaration of Helsinki and was approved by the Institutional Review Board of National Taiwan University Hospital. 


\section{Consent for publication \\ Not applicable.}

\section{Competing interests}

The authors declare that they have no competing interests.

\section{Author details}

${ }^{1}$ Division of Cardiology, Department of Internal Medicine, Camillian Saint Mary's Hospital Luodong, Yilan, Taiwan. ${ }^{2}$ Division of Cardiology, Department of Internal Medicine, National Taiwan University College of Medicine and Hospital, No.7, Chung Shan South Road, 100 Taipei, Taiwan. ${ }^{3}$ Graduate Institute of Epidemiology and Preventive Medicine, College of Public Health, National Taiwan University, Taipei, Taiwan. ${ }^{4}$ Department of Medical Research, National Taiwan University Hospital, Taipei, Taiwan.

Received: 2 June 2021 Accepted: 9 July 2021

Published online: 23 July 2021

\section{References}

1. Ahmadi SS, Svensson AM, Pivodic A, et al. Risk of atrial fibrillation in persons with type 2 diabetes and the excess risk in relation to glycaemic control and renal function: a Swedish cohort study. Cardiovasc Diabetol. 2020;19(1):9.

2. Aune D, Feng T, Schlesinger $S$, et al. Diabetes mellitus, blood glucose and the risk of atrial fbrillation: a system atic review and meta-analysis of cohort studies. J Diabet Complicat. 2018;32(5):501-11.

3. Jansen HJ, Bohne LJ, Gillis AM, et al. Atrial remodeling and atrial fibrillation in acquired forms of cardiovascular disease. Heart Rhythm. 2020;1:147-59.

4. Şerban RC, Scridon A. Data linking diabetes mellitus and atrial fibrillationhow strong is the evidence? From epidemiology and pathophysiology to therapeutic implications. Can J Cardiol. 2018;34(11):1492-502.

5. Torimoto K, Okada Y, Mori H, Tanaka Y. Relationship between fluctuations in glucose levels measured by continuous glucose monitoring and vascular endothelial dysfunction in type 2 diabetes mellitus. Cardiovasc Diabetol. 2013;12:1.

6. Saisho Y. Glycemic variability and oxidative stress: a link between diabetes and cardiovascular disease? Int J Mol Sci. 2014;15(10):18381-406.

7. Monnier L, Mas E, Ginet C, et al. Activation of oxidative stress by acute glucose fluctuations compared with sustained chronic hyperglycemia in patients with type 2 diabetes. JAMA. 2006;295(14):1681-7.

8. Jun JE, Jin SM, Baek J, et al. The association between glycemic variability and diabetic cardiovascular autonomic neuropathy in patients with type 2 diabetes. Cardiovasc Diabetol. 2015;14:70.

9. Gresele P, Guglielmini G, De Angelis M, et al. Acute, short-term hyperglycemia enhances shear stress-induced platelet activation in patients with type II diabetes mellitus. J Am Coll Cardiol. 2003;41(6):1013-20.

10. Zhaokun $\mathrm{Pu}$, Lai L, Yang $\mathrm{X}$, et al. Acute glycemic variability on admission predicts the prognosis in hospitalized patients with coronary artery disease: a meta-analysis. Endocrine. 2020;67(3):526-34.

11. Okada K, Hibi K, Gohbara M, et al. Association between blood glucose variability and coronary plaque instability in patients with acute coronary syndromes. Cardiovasc Diabetol. 2015;14:111.

12. Besch G, Pili-Floury S, Morel C, et al. Impact of post-procedural glycemic variability on cardiovascular morbidity and mortality after transcatheter aortic valve implantation: a post hoc cohort analysis. Cardiovasc Diabetol. 2019;18(1):27.

13. Zhang T, Gong Su, Mi S-H, et al. Association between blood glucose variability and the characteristics of vulnerable plaque in elderly non-ST segment elevation acute coronary syndrome patients. Int Heart J. 2019;60(3):569-76.

14. Feng W, Li Z, Guo W, et al. Association between fasting glucose variability in young adulthood and the progression of coronary artery calcification in middle age. Diabetes Care. 2020;43(10):2574-80.

15. Yokota S, Tanaka H, Mochizuki Y, et al. Association of glycemic variability with left ventricular diastolic function in type 2 diabetes mellitus. Cardiovasc Diabetol. 2019;18(1):166.

16. Echouffo-Tcheugui JB, Zhao S, Brock G, et al. Visit-to-visit glycemic variability and risks of cardiovascular events and all-cause mortality: the ALLHAT study. Diabetes Care. 2019;42(3):486-93.
17. Segar MW, Patel KV, Vaduganathan M, et al. Association of long-term change and variability in glycemia with risk of incident heart failure among patients with type 2 diabetes: a secondary analysis of the ACCORD trial. Diabetes Care. 2020;43(8):1920-8.

18. Bancks MP, Carnethon MR, Jacobs DR Jr, et al. Fasting glucose variability in young adulthood and cognitive function in middle age: the coronary artery risk development in young adults (CARDIA) study. Diabetes Care. 2018;41(12):2579-85.

19. Li S, Nemeth I, Donnelly L, et al. Visit-to-visit HbA 1c variability is associated with cardiovascular disease and microvascular complications in patients with newly diagnosed type 2 diabetes. Diabetes Care. 2020;43(2):426-32.

20. Hirakawa Y, Arima H, Zoungas S, et al. Impact of visit-to-visit glycemic variability on the risks of macrovascular and microvascular events and all-cause mortality in type 2 diabetes: the ADVANCE trial. Diabetes Care. 2014;37(8):2359-65.

21. Cardoso CRL, Leite NC, Moram CBM, et al. Long-term visit-to-visit glycemic variability as predictor of micro- and macrovascular complications in patients with type 2 diabetes: the Rio de Janeiro Type 2 Diabetes Cohort Study. Cardiovasc Diabetol. 2018;17(1):33.

22. Lee $\mathrm{S}-\mathrm{R}$, Choi E-K, Han K-D, et al. Effect of the variability of blood pressure, glucose level, total cholesterol level, and body mass index on the risk of atrial fibrillation in a healthy population. Heart Rhythm. 2020;17(1):12-9.

23. Bohne $L$, Jansen $H J$, Daniel I, et al. Electrical and structural remodeling contribute to atrial fibrillation in type 2 diabetic $\mathrm{db} / \mathrm{db}$ mice. Heart Rhythm. 2021;18(1):118-29.

24. Saito S, Teshima Y, Fukui A, et al. Glucose fluctuations increase the incidence of atrial fibrillation in diabetic rats. Cardiovasc Res. 2014;104(1):5-14.

25. Ying C, Liu T, Ling H, et al. Glucose variability aggravates cardiac fibrosis by altering AKT signalling path. Diab Vasc Dis Res. 2017;14(4):327-35.

26. Ravi R, Balasubramaniam V, Kuppusamy G, et al. Current concepts and clinical importance of glycemic variability. Diabetes Metab Syndr. 2021;15(2):627-36.

27. Fatemi O, Yuriditsky E, Tsioufis C, et al. Impact of intensive glycemic control on the incidence of atrial fibrillation and associated cardiovascular outcomes in patients with type 2 diabetes mellitus (from the Action to Control Cardiovascular Risk in Diabetes Study). Am J Cardiol. 2014;114(8):1217-22.

28. Wright RJ, Newby DE, Stirling D. Effects of acute insulin-induced hypoglycemia on indices of inflammation: putative mechanism for aggravating vascular disease in diabetes. Diabetes Care. 2010;33(7):1591-7.

29. Frier BM, Schernthaner G, Heller SR. Hypoglycemia and cardiovascular risks. Diabetes Care. 2011;34(Suppl 2):S132-7.

30. Ceriello A, Monnier L, Owens D. Glycaemic variability in diabetes: clinical and therapeutic implications. Lancet Diabetes Endocrinol. 2019;7(3):221-30.

31. Sheng C-S, Tian J, Miao Ya, et al. Prognostic significance of long-term HbA $1 \mathrm{c}$ variability for all-cause mortality in the ACCORD trial. Diabetes Care. 2020;43(6):1185-90

32. Jang J-Y, Moon S, Cho S, et al. Visit-to-visit HbA1C and glucose variability and the risks of macrovascular and microvascular events in the general population. Sci Rep. 2019;9(1):1374.

33. Yang C-Y, Pei-Fang Su, Hung J-Y, et al. Comparative predictive ability of visitto-visit HbA1c variability measures for microvascular disease risk in type 2 diabetes. Cardiovasc Diabetol. 2020;19(1):105.

34. Noyes JD, Soto-Pedre E, Donnelly LA, et al. Characteristics of people with high visit-to-visit glycaemic variability in Type 2 diabetes. Diabet Med. 2018;35(2):262-9.

35. Gumprecht J, Lip GYH, Sokal A, et al. Relationship between diabetes mellitus and atrial fibrillation prevalence in the Polish population: a report from the Non-invasive Monitoring for Early Detection of Atrial Fibrillation (NOMEDAF) prospective cross-sectional observational study. Cardiovasc Diabetol. 2021:20(1):128.

\section{Publisher's Note}

Springer Nature remains neutral with regard to jurisdictional claims in published maps and institutional affiliations. 